\title{
Análise comparativa do peso específico dos tratores agrícolas fabricados no Brasil e seus efeitos sobre a seleção e uso
}

\author{
Comparative analysis of the especific weight and its effects on the selection and use \\ of the brazilian wheeled tractors
}

José Fernando Schlosser $^{1}$ Henrique Debiasi ${ }^{2}$ Jorge Alex Willes ${ }^{2}$ Otávio Dias da C. Machado $^{2}$

\section{RESUMO}

$O$ peso do trator influi diretamente na recomendação e no seu desempenho a campo. Esta variável é, muitas vezes, característica do fabricante, induzindo a necessidade de informações comparativas entre os modelos. O objetivo deste trabalho foi estudar os tratores agrícolas brasileiros no que se refere ao peso, visando obter dados que auxiliem na seleção e no seu uso adequado. O levantamento englobou 103 modelos de tratores comerciais das marcas Massey Ferguson, Agrale, New Holland, John Deere, Ursus e Valtra. Foram analisados os parâmetros peso e relação peso/ potência, separadamente em diferentes marcas, tipo de tração e faixas de potência. A análise estatística foi feita através do teste $t$ de Student. Foram observadas diferenças significativas para classificação de marcas e faixas de potência no parâmetro relação peso/potência, diferindo também no critério tipo de tração quando analisado o peso. Além disso, verificou-se que a relação peso/potência sem lastro diminui com o aumento da potência. Portanto, os tratores agrícolas de rodas brasileiros são heterogêneos no que se refere ao peso e à relação peso potência, o que afeta o desempenho econômico dos mesmos, sendo importantes fatores a serem considerados na seleção $e$ no uso destas máquinas.

Palavras-chave: tratores, lastragem, eficiência.

\section{ABSTRACT}

The aptitude and performance of the agricultural tractors on field conditions have been influenced by the tractor weight. This parameter has varied mainly according to the manufacturers, generating a demand of comparative information. The objective of this research was to analyze the weight of Brazilian agricultural tractors, aiding in the selection and in its appropriate use. The present research included 103 models of commercial tractors of the brands Massey Ferguson, Agrale, New Holland, John Deere, Ursus and Valtra. Tractor weight and weight/power relationship, separately in different brands, traction type and power groups, were analyzed. The data were analyzed through the test (Student). Significant differences were observed for the brands and power groups when the weight/power relationship was considered. The parameter weight has differed also for the traction type. Besides, the weight/power relationship without ballast weight has decreased with the increase of power. Therefore, Brazilian agricultural wheeled tractors are heterogeneous regarding weight and weight/power, what influences their economic performance. These parameters must be considered during the use and choice of a tractor.

Key words: tractors, ballast, efficiency.

\section{INTRODUÇÃO}

A caracterização ponderal é a parte do ensaio de uma máquina agrícola que trata especificamente da caracterização e mensuração relativas ao peso do espécime considerado (MIALHE, 1996). O peso do trator influi diretamente no desempenho a campo deste (MÁRQUEZ, 1990), sendo importante também na definição da aptidão na execução de determinadas tarefas (BILLER \& OLFE, 1986; MÁRQUEZ, 1990; RENIUS, 1994; BIONDI et al., 1996; LINARES, 1996). Considerando estas referências, os

\footnotetext{
${ }^{1}$ Engenheiro Agrônomo, Professor Titular, Doutor, Departamento de Engenharia Rural, Centro de Ciências Rurais, Universidade Federal de Santa Maria, Campus Universitário Camobi, 97105-900, Santa Maria, RS, Brasil. E-mail: schlosse@ccr.ufsm.br. Autor para correspondência.

${ }^{2}$ Engenheiro Agrônomo, MSc.
} 
tratores mais leves, com relações peso/potência em torno dos $35 \mathrm{~kg} \mathrm{~kW}^{-1}$, são adequados à execução de operações mais leves e a maiores velocidades, tais como pulverização, transporte interno e externo à propriedade, entre outras. Já os tratores com relação peso/potência em torno dos $60 \mathrm{~kg} \mathrm{~kW}^{-1}$ são apropriados a operações de maior exigência de força de tração, desenvolvendo, portanto menores velocidades, tais como aração, escarificação, gradagem e outras.

A evolução histórica do peso do trator, bem como da relação peso/potência, vêm sendo estudada por diversos autores. Em geral, os tratores vêm se tornando mais leves, bem como a relação peso/potência vem sendo reduzida, sendo essa redução maior para tratores de potência mais elevada (LAPERUTA FILHO et al., 1989; MÁRQUEZ, 1990; RENIUS, 1994; BIONDI et al., 1996). Conforme MÁRQUEZ (1990) e BIONDI et al. (1996), a redução no peso dos tratores é explicada pelo melhor dimensionamento do chassi, que elimina materiais desnecessários e reduz os custos de produção. Esta redução provoca diminuição da resistência ao rolamento, com importante decréscimo da potência perdida, e induz ao uso implementos com engate no sistema hidráulico de três pontos, que proporcionam transferência de carga dinâmica às rodas motrizes, substituindo com vantagem o lastro fixo. A redução de peso imprime versatilidade ao trator, entretanto as operações que demandam grande esforço de tração determinam uma alta dependência de lastro.

Durante a fase de projeto de um trator agrícola, as características ponderais são determinadas pelas diferentes concepções dos fabricantes. Isto provoca a demanda de informações comparativas entre marcas e modelos sempre que for necessário selecionar estas máquinas. $\mathrm{O}$ objetivo deste trabalho foi estudar os tratores agrícolas fabricados e comercializados no Brasil, no que se refere ao peso, visando obter dados que auxiliem na seleção e no uso adequado destas máquinas.

\section{MATERIAL E MÉTODOS}

A análise englobou 106 modelos de tratores agrícolas comercializados pelas marcas Massey Ferguson, Agrale, New Holland, John Deere, Ursus e Valtra, das versões com tração simples $(4 \times 2)$ e tração dianteira auxiliar (TDA) em todas as faixas disponíveis no país. Para fins de diferenciação entre os modelos, foram utilizados os parâmetros peso do trator, sem lastro (kg) e relação peso/potência $\left(\mathrm{kg} \mathrm{kW}^{-1}\right)$. Os dados para a comparação foram informados pelos fabricantes.
Para fins de simplificação e pelo uso popular, o termo peso será utilizado como sinônimo de massa do veículo. $\mathrm{Na}$ análise estatística dos dados, foi empregado o teste $\mathrm{t}$ de Student, a 5\% de probabilidade de erro. Já as regressões foram efetuadas através do programa informático MICROSOFT EXCEL ${ }^{\circledR}$.

\section{RESULTADOS E DISCUSSÃO}

Foram comparados 106 tratores, sendo 63 com tração 4x2 e 43 tratores com tração do tipo TDA, verificando-se que, segundo o tipo de tração, conforme mostra a tabela 1 , verificam-se diferenças significativas entre as marcas de tratores quanto ao peso, potência e relação peso/potência. Nota-se que, em uma análise geral de todas as marcas e todas as faixas de potência, entre os tratores equipados com tração simples não houve diferença significativa para os tratores Massey Ferguson, John Deere e Valtra, já nos modelos com tração dianteira auxiliar as diferenças não foram significativas entre Massey Ferguson, New Holland e John Deere para a mesma variável. Analisando-se a tabela 1, verifica-se que a relação peso/potência (kg $\mathrm{kW}^{-1}$ ) não foi diferente entre os tipos de tração (TDA ou 4x2). Para os tratores de tração simples, esta relação foi maior e não diferiu significativamente entre os modelos de tratores das marcas Agrale e Ursus e as menores relações foram as dos tratores New Holland. Para os tratores de tração dianteira auxiliar, as maiores relações peso/potência foram para os tratores Agrale e as menores para os tratores New Holland, não havendo diferença para as marcas John Deere, Ursus e Valtra.

Analisando-se conjuntamente as tabelas 1 e 2, nota-se que o parâmetro peso também refletiu em diferenças quando os tratores analisados foram comparados segundo a marca. Conforme mostra a tabela 2, as marcas cujos modelos caracterizam-se pelos maiores valores de peso, foram MasseyFerguson, Valtra e John Deere, sendo que não houve diferença entre as mesmas. Os tratores da marca Agrale, por sua vez, apresentaram os menores pesos, em torno dos $2500 \mathrm{~kg}$, o que representa cerca de $60 \%$ a menos que os modelos de maior peso. Os tratores das marcas New Holland apresentaram um comportamento intermediário, possuindo em média um peso de $3500 \mathrm{~kg}$. Novamente, verifica-se que, à despeito do comportamento obtido em função da análise exclusiva do peso, quando se considera a relação peso/potência, nota-se uma semelhança entre os modelos produzidos pelas diferentes fábricas. Os tratores pertencentes às marcas Agrale e Ursus, não obstante, possuírem o menor peso, têm potências também menores 
Tabela 1 - Comparação entre as médias do peso e da relação peso/potência segundo o tipo de tração dos tratores agrícolas fabricados e comercializados no Brasil.

\begin{tabular}{|c|c|c|c|c|}
\hline Tração & Unidades & Peso (kg) & Potência (kW) & Relação peso/potência $\left(\mathrm{kg} \mathrm{kW}^{-1}\right)$ \\
\hline \multicolumn{5}{|c|}{$4 \times 2$} \\
\hline Média & 63 & $2.978,9^{\text {de }}$ & $52,75^{\mathrm{e}}$ & $58,08^{\text {cd }}$ \\
\hline Máximo & 1 & $7.336,0^{\mathrm{a}}$ & $140,00^{\mathrm{a}}$ & $87,57^{\mathrm{a}}$ \\
\hline Mínimo & 1 & $2.390,0^{\mathrm{e}}$ & $34,60^{\mathrm{f}}$ & $33,12^{\mathrm{f}}$ \\
\hline Massey Ferguson & 21 & $4.303,6^{\mathrm{b}}$ & $72,60^{\mathrm{d}}$ & $61,80^{\mathrm{bc}}$ \\
\hline Agrale & 4 & $2.820,0^{\text {de }}$ & $44,12^{\mathrm{e}}$ & $64,29^{\mathrm{b}}$ \\
\hline New Holland & 11 & $3.509,9^{c}$ & $80,67^{\mathrm{bc}}$ & $45,96^{\mathrm{e}}$ \\
\hline John Deere & 9 & $4.371,1^{b}$ & $74,04^{\mathrm{cd}}$ & $59,44^{\mathrm{cd}}$ \\
\hline Ursus & 4 & $3.332,5^{\mathrm{cd}}$ & $50,18^{\mathrm{e}}$ & $68,77^{\mathrm{b}}$ \\
\hline Valtra & 14 & $4.842,9^{b}$ & $87,38^{\mathrm{b}}$ & $57,25^{\mathrm{d}}$ \\
\hline \multicolumn{5}{|c|}{ TDA } \\
\hline Média & 43 & $4.227,4^{\mathrm{b}}$ & $76,64^{\mathrm{b}}$ & $57,96^{\mathrm{cd}}$ \\
\hline Máximo & 1 & $5200,0^{\mathrm{a}}$ & $93,00^{\mathrm{a}}$ & $83,87^{\mathrm{a}}$ \\
\hline Mínimo & 1 & $1050,0^{f}$ & $13,20^{f}$ & $43,10^{\mathrm{e}}$ \\
\hline Massey Ferguson & 16 & $3565,9^{c}$ & $60,57^{\mathrm{c}}$ & $60,66^{\mathrm{bc}}$ \\
\hline Agrale & 7 & $2000,7^{\mathrm{e}}$ & $33,34^{\mathrm{e}}$ & $63,03^{b}$ \\
\hline New Holland & 9 & $3509,9^{c}$ & $80,67^{\mathrm{b}}$ & $45,96^{\mathrm{e}}$ \\
\hline John Deere & 2 & $3342,5^{\mathrm{c}}$ & $59,11^{\mathrm{c}}$ & $56,76^{\text {cd }}$ \\
\hline Ursus & 6 & $2575,0^{\text {cd }}$ & $44,99^{d}$ & $57,76^{\mathrm{cd}}$ \\
\hline Valtra & 3 & $2946,67^{\text {cd }}$ & $52,27^{\mathrm{C}}$ & $56,15^{\mathrm{cd}}$ \\
\hline Total & 106 & & & \\
\hline
\end{tabular}

Médias seguidas por mesma letra não diferem significativamente pelo teste de Tukey a 5\%.

comparativamente aos tratores fabricados pela MF, John Deere e Valtra (Tabela 1 e 2), de modo que a relação peso/potência não diferiu entre as referidas marcas, ficando em torno dos $60 \mathrm{~kg} \mathrm{~kW}^{-1}$. Entretanto, para os modelos da marca New Holland, a relação peso/ potência foi menor, pois, apesar de apresentar níveis de potência semelhante aos modelos da Massey Ferguson, John Deere e Valtra, eles podem ser considerados construtivamente mais leves.

Quando o peso dos tratores nacionais em comercialização atualmente no Brasil é comparado conforme as faixas de potência observa-se, segundo expõe a tabela 3 , um crescimento no peso em função do aumento da potência. Os tratores com potência superior a $100 \mathrm{~kW}$ apresentaram um peso $(5.815 \mathrm{~kg})$ cerca de $120 \%$ superior ao peso dos tratores com potência inferior a 50kW. À semelhança do comportamento apresentado pelos modelos em função do tipo de tração e fabricante, a tendência muda quando a comparação é feita tendo-se como parâmetro a interação entre o peso e a potência. A relação peso/ potência diminuiu com o aumento da potência dos tratores. Para os modelos com mais de $100 \mathrm{~kW}$ de potência no motor, esta relação foi 38\% menor relativamente aos tratores equipados com motores de potência inferior a $50 \mathrm{~kW}$. Assim, pode-se inferir que o aumento na potência é proporcionalmente maior que o aumento do peso.

A figura 1 mostra o comportamento da relação peso/potência dos tratores nacionais em comercialização no Brasil segundo a potência nominal no motor, separadamente para os tratores 4x2 e TDA. Embora a dispersão dos dados seja grande, pode-se verificar claramente a tendência de decréscimo da relação peso/potência com o aumento da potência do motor, tanto para os tratores $4 \times 2\left(\mathrm{R}^{2}=0,71\right)$ quanto para os tratores TDA $\left(\mathrm{R}^{2}=0,69\right)$. Além disso, este decréscimo é mais acentuado para os tratores $4 \times 2$, que apresenta um coeficiente de inclinação da reta maior. Este comportamento, apresentado pelos tratores fabricados e comercializados no Brasil, confirma a tendência relatada por diversos autores (MÁRQUEZ, 1990; RENIUS, 1994; BIONDI et al., 1996) para os tratores comercializados em países pertencentes à Comunidade Econômica Européia. 
Tabela 2 - Comparação entre as médias do peso e da relação peso/potência segundo as diferentes marcas de tratores agrícolas fabricados e comercializados no Brasil.

\begin{tabular}{|c|c|c|c|c|c|c|}
\hline \multirow{2}{*}{ MARCA } & \multicolumn{6}{|c|}{ Significância $^{1}$} \\
\hline & MF & Agrale & New Holland & John Deere & Ursus & Valtra \\
\hline & \multicolumn{6}{|c|}{ Peso, (kg) } \\
\hline Massey Ferguson & - & $*$ & $*$ & ns & $*$ & ns \\
\hline Agrale & $*$ & - & $*$ & $*$ & $*$ & $*$ \\
\hline New Holland & $*$ & $*$ & - & $*$ & ns & $*$ \\
\hline John Deere & ns & $*$ & $*$ & - & $*$ & ns \\
\hline Ursus & $*$ & $*$ & ns & $*$ & - & $*$ \\
\hline \multirow[t]{2}{*}{ Valtra } & ns & $*$ & $*$ & ns & $*$ & - \\
\hline & \multicolumn{6}{|c|}{ Potência, (kW) } \\
\hline Massey Ferguson & - & $*$ & ns & ns & $*$ & ns \\
\hline Agrale & * & - & $*$ & $*$ & ns & $*$ \\
\hline New Holland & ns & $*$ & - & ns & $*$ & ns \\
\hline John Deere & ns & $*$ & ns & - & $*$ & ns \\
\hline Ursus & $*$ & ns & $*$ & $*$ & - & $*$ \\
\hline \multirow[t]{2}{*}{ Valtra } & ns & $*$ & ns & ns & $*$ & - \\
\hline & \multicolumn{6}{|c|}{ Relação peso/potência, (kg/kW) } \\
\hline Massey Ferguson & - & Ns & $*$ & Ns & Ns & Ns \\
\hline Agrale & ns & - & $*$ & Ns & Ns & Ns \\
\hline New Holland & $*$ & $*$ & - & $*$ & $*$ & $*$ \\
\hline John Deere & Ns & Ns & $*$ & - & Ns & Ns \\
\hline Ursus & Ns & ns & $*$ & ns & - & ns \\
\hline Valtra & Ns & ns & $*$ & ns & ns & - \\
\hline
\end{tabular}

* - Contraste entre duas marcas significativo pelo teste t de Student a 5\% de probabilidade de erro.

ns - Contraste entre duas marcas não significativo pelo teste t de Student a 5\% de probabilidade de erro.

- Contraste entre marcas iguais.

Pode-se deduzir que a relação peso/ potência dos tratores agrícolas fabricados e comercializados no Brasil varia, basicamente, em função da marca do trator e da potência do motor. É importante salientar que estes dados constituem-se em parâmetros que auxiliam na seleção dos tratores e na otimização do uso dos mesmos, visando à redução dos custos inerentes a maquinaria agrícola.

Os tratores agrícolas brasileiros de menor potência possuem relações peso/potência em torno dos $60 \mathrm{~kg} \mathrm{~kW}^{-1}$, conforme mostram a tabela $3 \mathrm{e} \mathrm{a} \mathrm{figura}$ 1. Estes dados confirmam o que relatam RENIUS (1994) e BILLER \& OLFE (1986), que indicam que os tratores de menor potência são comumente utilizados para trabalhos leves, pode-se considerar que a relação peso/potência de $60 \mathrm{~kg} \mathrm{~kW}^{-1}$ é bastante elevada comparativamente aos $35 \mathrm{~kg} \mathrm{~kW}^{-1}$ considerado ideal para operações leves por MÁRQUEZ (1990), BIONDI et al. (1996) e LINARES (1996). Portanto, estes tratores pequenos, quando executam operações leves, perdem potência e energia para vencer a resistência ao rolamento adicional ocasionada pelo excesso de peso, o que resulta em diversos prejuízos, tais como maior consumo de combustível, aumento da compactação do solo e menor capacidade operacional.

Levando-se em consideração que, para o agricultor, não é possível diminuir essa relação, pois não há como retirar peso, a minimização desse problema deve ser buscada por ocasião da compra do trator. Analisando-se novamente a figura 1, observa-se que, para uma mesma potência, há uma grande variação na relação peso/potência podendo-se encontrar tratores de $40 \mathrm{~kW}$ com relações peso/potência que variam de aproximadamente 50 a $90 \mathrm{~kg} \mathrm{~kW}^{-1}$. Assim, conforme MÁRQUEZ (1990), é importante, neste caso, escolher o modelo com menor relação peso/potência compatível com a natureza do trabalho a ser efetuado, buscando melhores resultados. 
Tabela 3 - Comparação entre as médias de peso e da relação peso/potência segundo as diferentes faixas de potência dos tratores agrícolas fabricados e comercializados no Brasil.

\begin{tabular}{lcccc}
\hline \multirow{2}{*}{$\begin{array}{c}\text { Faixa de } \\
\text { potência }(\mathrm{kW})\end{array}$} & Média & \multicolumn{4}{c}{ Significância $^{1}$} \\
\cline { 3 - 5 } & \multicolumn{5}{c}{ Peso $(\mathrm{kg})$} \\
\hline$<50$ & $2.649,80$ & - & $*$ & $*$ \\
\hline 50 a 100 & $3.740,10$ & $*$ & - & $*$ \\
\hline$>100$ & $5.815,60$ & $*$ & - & - \\
\hline & Relação peso/potência $\left(\mathrm{kg} \mathrm{kW}^{-1}\right)$ & \\
\hline$<50$ & 66,37 & - & $*$ & $*$ \\
\hline 50 a 100 & 55,96 & $*$ & - & $*$ \\
\hline$>100$ & 48,05 & $*$ & - & - \\
\hline
\end{tabular}

* - Contraste entre duas marcas significativo pelo teste $t$ de Student a 5\% de probabilidade de erro.

ns - Contraste entre duas marcas não significativo pelo teste t de Student a 5\% de probabilidade de erro.

- Contraste entre fixas iguais.
Nota-se que a tendência apresentada pelos tratores de menor potência inverte-se quando são analisados os tratores de maior potência (acima de 80kW). Neste caso, são encontrados modelos com relações peso/potência que variam de 30 a $65 \mathrm{~kg} \mathrm{~kW}^{-1}$. Estes últimos $\left(65 \mathrm{~kg} \mathrm{~kW}^{-1}\right)$ somente podem ser utilizados eficientemente para a execução de operações pesadas, com grande demanda de força de tração, impossibilitando rendimentos semelhantes em operações leves. Já os modelos com mais de $80 \mathrm{~kW}$ e com relações peso/potência mais próximas de $30 \mathrm{~kg}$ $\mathrm{kW}^{-1}$ são mais versáteis, podendo ser utilizados tanto para operações mais leves quanto para operações mais pesadas. Isto traz como vantagem a diminuição do número de tratores necessários na propriedade, o que reduz significativamente os custos, porém aumenta a dependência da adição de peso (lastragem) quando empregados para a execução de trabalhos mais pesados.

Com relação ao uso de tratores de potência elevada e com baixa relação peso/potência, para a

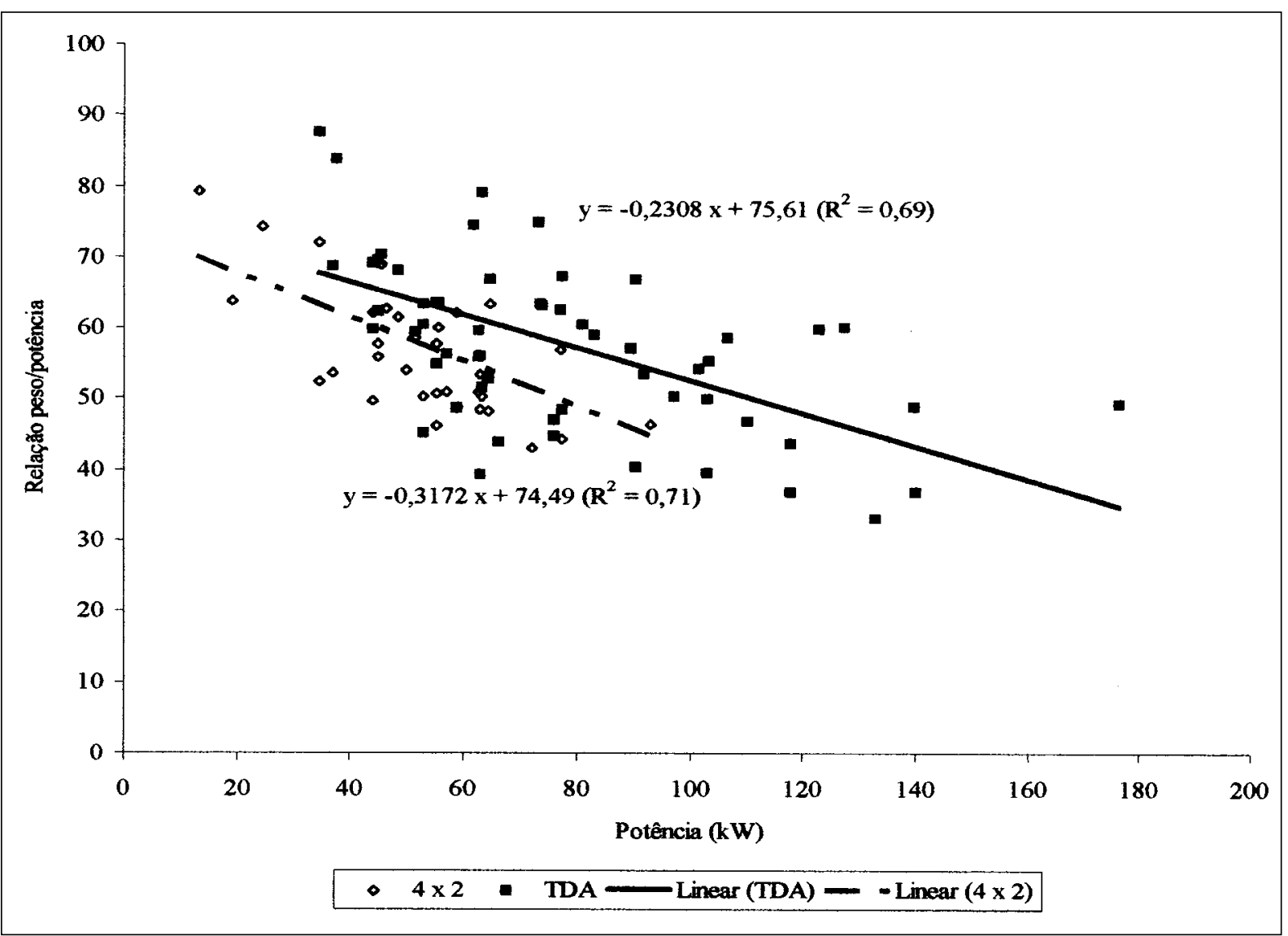

Figura 1 - Comportamento da relação peso/potência segundo a potência nominal dos tratores agrícolas fabricados e comercializados no Brasil.

Ciência Rural, v.35, n.1, jan-fev, 2005. 
execução de trabalhos pesados, é necessário considerar dois fatores agravantes. Como esses tratores são mais dependentes de lastro, há necessidade de uma maior capacitação de seus usuários, para que a adição de peso seja feita de maneira correta. Via de regra, no Brasil, o nível de formação dos usuários é baixo, dificultando a eficiente utilização dos tratores com essas características. É comum o agricultor adquirir um trator de grande potência e baixa relação peso/potência, e subutilizá-lo por não lastrá-lo corretamente e não adequar a velocidade de trabalho. Isto se reflete diretamente no incremento dos custos fixos e variáveis da máquina, em função da subutilização e do desperdício de combustível. Como não é possível aumentar o peso, para que haja aproveitamento adequado da potência do motor, é necessário o aumento da velocidade de trabalho, o que muitas vezes não é possível devido à natureza da operação que está sendo efetuada.

Alguns modelos de baixa potência podem ter sua relação peso/potência diminuída grandemente, pois modelos de potência similar, conforme mostra a figura 1 , apresentam valores bem menores para a referida relação.

\section{CONCLUSÕES}

As características, heterogêneas no universo analisado, delimitaram diferentes aptidões e usos para os tratores, de certa forma relacionadas com o nível de potência ao qual se enquadram, o que determina a seleção de um trator para uma dada atividade.

Os tratores fabricados, no Brasil, apresentam clara tendência em diminuir a relação peso/ potência com o aumento da potência bruta no motor, nas duas versões analisadas.
Verifica-se uma certa inadequação dos projetos dos tratores agrícolas fabricados e comercializados no Brasil, principalmente considerando os tratores de menor potência. No que se refere aos tratores de maior potência, que devem apresentar uma maior versatilidade, há uma maior dependência de lastragem e necessidade de um aumento na quantidade de lastro possível de ser adicionada, juntamente ao desenvolvimento de mecanismos que dinamizem este procedimento.

\section{REFERÊNCIAS BIBLIOGRÁFICAS}

BILLER, R.H.; OLFE, G. Collect of data on tractor use by questionnaires and by eletronical data approval. Journal of Agricultural Engineering Research, v.34. p.219-227, 1986.

BIONDI, P. et al. Technical trends of tractors and combines (1960-1989) based on Italian type-approval data. Journal of Agricultural Engineering Research, v.65, p.1-14, 1996.

LAPERUTA FILHO, J. et al. Evolução do peso dos tratores agrícolas ensaiados no Brasil. In: CONGRESSO BRASILEIRO DE ENGENHARIA AGRÍCOLA, 18., 1989, Recife. Resumo... Recife : Universidade Federal Rural de Pernambuco, 1989. p.13.

LINARES, P. Teoría de la tracción de tractores agrícolas. Madrid : ETSIA, 1996. 157p. (Apuntes Didácticos Departamento de Publicaciones de la Escuela Técnica Superior de Ingenieros Agrónomos).

MÁRQUEZ, L. Solo Tractor '90. Madrid : Laboreo, 1990. 198p. (Apuntes didácticos).

MIALHE, L.G. Máquinas agrícolas: ensaios \& certificação. São Paulo : She Kinah, 1996. 721p.

RENIUS, K. Th. Trends in tractor design with particular reference to europe. Journal of Agricultural Engineering Research, v.57, p.3-22, 1994. 